\title{
Ein selbstbestimmtes Leben für alle. Gesellschaftliche Voraussetzungen von Autonomie
}

\section{Martin Kronauer Günther Schmid}

Sein Leben selbstbestimmt führen zu können, setzt soziale Sicherheiten voraus, die es erlauben, längerfristig zu planen, im eigenen Lebensverlauf erstrebenswerte Ziele zu verfolgen und nicht nur aus Zwängen heraus zu handeln. Derartige Absicherungen sind unverzichtbare Bestandteile sozialer Grundrechte. Auf ihnen bauen zivilgesellschaftliche und politische Beteiligungsmöglichkeiten auf. In demokratischen Gesellschaften müssen sie für alle Mitglieder des politischen Gemeinwesens verfügbar sein, was wiederum grundlegende Gleichheit im Anspruch auf Sicherheiten vermittelnde Ressourcen und Verfahren einschließt. Autonomie fördernde Sicherheit und Gleichheit sind schließlich auch die Voraussetzungen für die nachhaltige Steigerung der Lebensqualität einer Gesellschaft insgesamt. Dies legt nicht nur der moderne Diskurs normativer und empirischer Gerechtigkeitsvorstellungen nahe, wie im Folgenden gezeigt werden soll, sondern wird auch durch die weiteren Beiträge dieses Schwerpunkthefts bestätigt.

\section{Autonomie braucht Gleichheit und Sicherheit}

\subsection{DIE WIEDERKEHR DES ELITISMUS}

Gegen die Verbindung von Sicherheit, Gleichheit und Autonomie liefen immer schon elitär-konservative Denkströmungen einerseits und marktliberal orientierte andererseits Sturm. Freiheit würde durch soziale Sicherheiten erstickt, individuelle Entfaltung durch Gleichheit („Gleichmacherei“) behindert, das Mittelmaß herrsche über das Vorzügliche und Bahnbrechende. In den letzten Jahrzehnten haben diese Strömungen wieder an Boden, wenn nicht gar die Oberhand in den gesellschaftspolitischen Debatten gewonnen. Das Debakel an den Finanzmärkten, die (vorübergehende?) Rettung des Bankensystems durch Staatsinterventionen (materiell: durch die Steuerzahler) schien solchem Argumentieren die Luft zu nehmen. Die Halbherzigkeiten der Interventionen jedoch, die fehlende Perspektive einer Wiedergewinnung gesellschaftlicher Regelungshoheit über die Märkte haben die Kräfteverhältnisse in der Debatte sogleich wieder verändert. Die marktliberale Strömung wurde, von den Wählern gestärkt, in die Regierung geschickt. Vorreiter des neuen Elitismus testen aus, wie weit sie im argumentativen Kampf für die Ausweitung von Ungleichheit gehen können und stoßen (trotz offiziell noch immer aufgerichteter Tabus) auf erhebliche Resonanz gerade in den sogenannten gebildeten Schichten. ${ }^{1}$ Dabei glänzt die selbst ernannte Denkelite vor allem durch Ignoranz gegenüber der langen Geschichte theoretischer Begründungen und empirischer Untersuchungen von sozialstaatlichem Handeln. Was diese „Klassenkämpfer von oben“ befeuert, sind aristokratisches Gehabe ${ }^{2}$ und das Schielen auf den eigenen Kontostand. ${ }^{3}$

1 Vgl. etwa Karl-Heinz Bohrers nietzscheanische Abkanzlung der Plebs im Kontext der HonnethSloterdijk Kontroverse oder Thilo Sarrazins (2009) Invektiven gegen die Unterschicht und die Massenuniversität, verbunden mit dem Plädoyer für ein Berlin der Eliten; von seinem „Bestseller “ ganz zu schweigen.

2 So versteigt sich Sloterdijk bei seinem Plädoyer für eine Umstellung von „Enteignung" durch Steuern auf "Gabe“ zu der Wortwahl, erst durch eine solche Veränderung könne die Wende von einer "gierbeherrschten“ zu einer "stolzbewegten“ Gesellschaftsform bewirkt werden, von der - wie Sloterdijk meint - so viele Kritiker der bestehenden Verhältnisse "gerade auch im linken Spektrum" träumten. Und dann noch die kokette rhetorische Frage: „Was soll überhaupt aus einer Linken werden, die exklusiv an den Begriffen ,Enteignung' und, Besteuerung ' klebt und der zu einer Ethik der Gabe schlechterdings nichts einfällt?" (Sloterdijk 2009).
Gefährlich im Hinblick auf die $\mathrm{Zu}$ kunft der Demokratie und ihres sozialmateriellen Unterbaus sind diese Strömungen vor allem deshalb, weil sie derzeit weitgehend verunsicherte Mittelklassen ansprechen, und dies in einer Periode, in

3 Der emeritierte und somit aus Steuergeldern im Rahmen einer sozialstaatlichen Leistung entlohnte Professor für Neuere deutsche Literaturgeschichte und Ästhetik Karl-Heinz Bohrer klagt, den Kritikern von Sloterdijk gehe es um nichts anderes als um die „Verteidigung des allmählich verkommenden Sozialstaats“, der ihn persönlich und viele andere „um die Pfründe wohlverdienten finanziellen Zugewinns " gebracht habe, indem er ihm „die Hälfte der beträchtlichen Summen für akademische Auszeichnungen oder für Kulturpreise" (Bohrer 2009) wieder abgenommen habe (was ihm als "gezielte Bestrafung von Individuen" gilt - die Verf.).

Martin Kronauer, Dr. phil., ist Professor für Strukturwandel und Wohlfahrtsstaat in internationaler Perspektive an der Hochschule für Wirtschaft und Recht (HWR) Berlin.

e-mail: kronauer@hwr-berlin.de Günther Schmid, Dr. phil., ist Prof. em. für Ökonomische Theorie der Politik an der Freien Universität Berlin und Direktor em. der Abteilung Arbeitsmarktpolitik und Beschäftigung am Wissenschaftszentrum Berlin für Sozialforschung (WZB). e-mail: gues@guentherschmid.de 
der heftige Auseinandersetzungen darüber stattfinden, wie die gesellschaftlichen Kosten der Krise zu verteilen sind. Ob sich die Mehrheit der Angehörigen dieser Mittelklassen nach wie vor den kollektiven Sicherungssystemen verpflichtet fühlen wird, aus denen sie bislang am meisten Nutzen zog, oder ob sie eine Monopolisierung von Ressourcen (vor allem in den Bereichen Bildung und Krankenversorgung) auf Kosten benachteiligter Gruppen unterstützen wird, scheint noch nicht entschieden.

\subsection{EINE NOTWENDIGE KRITIK}

In dieser kritischen Periode setzt sich das vorliegende Schwerpunktheft zum Ziel, notwendige Verbindungen zwischen Gleichheit, Sicherheit und Autonomie im gegenwärtigen flexiblen Kapitalismus theoretisch und empirisch auszuloten. Vor allem wird die Behauptung, Steuern und Abgaben seien auf die Stufe von „Diebstahl“ oder „Enteignung“ zu stellen und würden Freiheit und Autonomie von Menschen in unwürdiger Form begrenzen oder gar strangulieren, als falsch, wenn nicht gar als absurd zurückzuweisen sein (siehe Reiners in diesem Heft). Wer beispielsweise dem arbeitenden Volk und nicht der ,intellektuellen Plebs“" aufs Maul schaut, stellt fest, dass der Anteil derjenigen, die ihr Nettoerwerbseinkommen als ungerecht ansehen, mit steigender Höhe des Bruttostundenlohns von etwa $50 \%$ (unterstes Fünftel 2007) auf $23 \%$ (oberstes Fünftel) abnimmt - trotz des progressiven Steuertarifs (Becker/Hauser 2009, S. 50).

Ebenso entbehrt die These, Freiheit sei unvermeidlich mit Ungleichheit verbunden, ja Ungleichheit überhaupt sei die Triebfeder einer dynamischen Gesellschaft, jeder empirischen Grundlage. Es ist, wie sich in diesem und anderen Beiträgen herausstellen wird, genau im Gegenteil die ungleiche Verteilung von Einkommen und Lebenschancen, die sowohl eine kreative Dynamik gesellschaftlicher Entwicklung als auch die Verwirklichung autonom entschiedener Lebensentwürfe abwürgt (Wilkinson/Pickett 2009; Böhnke in diesem Heft). Das gilt insbesondere für die Ungleichheit der Bildungschancen, welche die große Mehrheit junger Menschen beispielsweise vom Wettbewerb „akademischer Auszeichnungen “von vornherein ausschließt (siehe den Beitrag von Hopf in diesem Heft).
In diesem, das vorliegende Schwerpunktheft einleitenden Beitrag umreißen wir zunächst das Verhältnis von Gleichheit, Sicherheit und Autonomie im Rahmen der normativen wissenschaftlichen Debatte um gerechtigkeits- und wohlfahrtstheoretische Grundlagen des modernen Sozialstaats (Abschnitt 2). In einem weiteren Schritt (Abschnitt 3) ergänzen wir die Argumentation um eine Diskussion über empirisch vorzufindende Gerechtigkeitsvorstellungen und deren Verhältnis zum Anspruch auf ein selbstbestimmtes Leben. Abschnitt 4 bilanziert den Handlungsbedarf. Die folgenden Beiträge dieses Heftes reflektieren theoretisch und empirisch jeweils unterschiedliche Aspekte der hier aufgezeigten notwendigen Verbindung von Sicherheit, Gleichheit und Autonomie.

\section{2 \\ Gleichheit, Sicherheit und Autonomie als Elemente einer gerechten Gesell- schaftsordnung}

Das oberflächlich sympathisch klingende Ansinnen von Sloterdijk, mehr Freiwilligkeit und damit Eigenverantwortung bei der Herstellung von Gemeinschaftsgütern oder beim solidarischen Einkommensausgleich zu fordern, ist weder durch verhaltenstheoretische noch durch normativ-analytische Grundprinzipien gedeckt. Es löst nicht die große Frage, unter welchen materiellen, kognitiven und psychologischen Voraussetzungen die Übertragung von mehr Eigenverantwortung, etwa bei der Bewältigung von Risiken im Lebenslauf, gerechtfertigt wäre. Wie könnte eine gerechte Verantwortungsteilung zwischen Individuum und Gesellschaft überhaupt aussehen? Das ist eine komplizierte, hier nicht erschöpfend zu behandelnde Frage, zumal in der aktuellen sozialphilosophischen Debatte das Problem gerechter Verantwortungsteilung als noch weitgehend ungelöst betrachtet wird (Arneson 2010). Im Folgenden sollen die wichtigsten Überlegungen an anderer Stelle (u.a. Schmid 2008, 2011) aufgegriffen und fortgeführt werden, wobei wir uns zunächst auf den normativ-analytischen Diskurs von Gleichheit und Gerechtigkeit konzentrieren.

Unter dem Aspekt der Autonomie stellt sich vor allem die Frage, wie die Verantwortungsteilung zwischen Individuum und Gesellschaft erfolgen soll. Aus dieser Perspektive eignet sich weder die utilitaristische Wohlfahrtstheorie, noch reicht die rationale Vertragstheorie von John Rawls (1990) als Grundlage für die Entwicklung normativer Prinzipien aus. Zur gängigen Theorie der Gerechtigkeit als Chancengleichheit (Fairness) ist die Theorie der Gerechtigkeit als Ressourcengleichheit (Solidarität) des amerikanischen Rechtsphilosophen Ronald Dworkin (2000) als weiterer Ausgangspunkt hinzuzunehmen. Nur die dieser Theorie zugrunde liegende Verantwortungsethik kann dem Begriff individueller wie gesellschaftlicher Solidarität ein klares normatives Fundament geben. Die Debatte um Ressourcengleichheit endet jedoch schnell in unlösbaren Konflikten, wenn keine Kriterien genannt werden, wann und in welcher Hinsicht von „gleichen Ressourcen“ die Rede sein soll (Roemer 2009). Die Theorie der Befähigungsgerechtigkeit von Amartya Sen (2009), d.h. der Möglichkeit, die verfügbaren Ressourcen selbstbestimmt (autonom) zur Verwirklichung von Lebensplänen (Freiheit) nutzen zu können, also gleichsam Autor des eigenen Lebens zu sein, ist ein vielversprechender Weg, um solchen Konflikten aus dem Wege zu gehen. Schließlich muss auch immer die Reichweite (Inklusion) der geltenden Gerechtigkeitsprinzipien hinterfragt werden. In welchem sozialen und geografischen Raum soll Gerechtigkeit gelten? Nur lokal, oder auch global? Wer gehört zur „Schicksalsgemeinschaft"? Obwohl dazu kaum etablierte Theorien verfügbar sind, müssen gesellschaftliche Ordnungen immer auch den Test des Gerechtigkeitsprinzips sozialer Inklusion bestehen.

\subsection{CHANCENGLEICHHEIT}

John Rawls (1990, 2001) leitet die allgemein einvernehmlichen Kriterien für Gerechtigkeit von einer konstruierten Vertragstheorie ab. Hinter dem „Schleier des Unwissens" würden sich die meisten für das sogenannte Differenzprinzip entscheiden. Ökonomische Ungleichheit sei in einer freien Gesellschaft unvermeidlich, aber im Hinblick auf gleiche Ausstattung von Primärgütern wie das Recht auf körperliche Unverletzlichkeit, auf Wohnung, Nahrung und Wasser sowie das Recht auf Meinungsfreiheit oder freie Berufswahl dürfe es keine Differenz geben. Ansonsten seien Ungleichheiten (Differenzen) 
gerechtfertigt, solange sie die Position der ökonomisch am schlechtesten gestellten Gruppe verbessern. Damit plädiert Rawls für die Maximierung des Minimums (Maximin-Prinzip), während die Wohlfahrtstheoretiker oder Utilitaristen für die Maximierung des Durchschnitts eintreten („das größte Glück der größten Zahl“).

Die Unterschiede der beiden Strategien (Maximierung des Minimums und Maximierung des Durchschnitts) lassen sich an einem einfachen Rechenbeispiel veranschaulichen. Der Ausgangspunkt der Verteilung sei ein durchschnittliches Einkommen von 1.000 Einheiten, das niedrigste Quintil verfügt über 200, das höchste über 2.000 Einheiten, also eine Relation von 1:10. Angenommen, die Wohlfahrt und Verteilung entwickeln sich im Durchschnitt auf 1.500 Werteinheiten, das niedrigste Quintil verfügt wieder über 200, das höchste über 3.000 Einheiten, Relation 1:15; diese Verteilung wäre für die Wohlfahrtstheoretiker, aber nicht für Rawls akzeptabel. Angenommen, der Durchschnitt entwickelt sich nur auf 1.200, aber das niedrigste Quintil verbessert sich auf 300, das höchste auf nur 2.000 Einheiten, Relation 1:7; diese Verteilung wäre nun für Rawls, aber nicht für die Wohlfahrtstheoretiker akzeptabel.

\subsection{RESSOURCENGLEICHHEIT}

Ronald Dworkin (2000) hält Rawls' Vertragskonstruktion aus ethischen Gründen für problematisch. Sie unterscheide nicht, ob die Ungleichheit zufallsbedingt und extern verursacht, oder ob sie das Ergebnis einer persönlich freien Wahlentscheidung sei. Er plädiert daher für eine Gerechtigkeitstheorie, die auf den ethischen Grundprinzipien individueller Verantwortung und gesellschaftlicher Solidarität beruht, d.h. auf einer ethisch akzeptierten Balance von individuellen Rechten und Pflichten. Gerecht sei eine Verteilung, wenn sie die Umstände ungleicher Ergebnisse des Lebensstandards und der Lebensqualität berücksichtigt.

Danach können die Menschen nicht für Verteilungen verantwortlich gemacht werden, die durch externe Faktoren verursacht sind. Solche Faktoren, die individuell nicht beeinflusst werden können, sind angeborene Talente, Behinderungen und Anfälligkeit für Krankheiten oder externe Gegebenheiten wie Wetter und natürliche Umgebung. Dazu gehören aber auch die Zu- fälle des Marktes. Diese können bewirken, dass Menschen mit denselben Ausbildungsniveaus und mit denselben Anstrengungen durchaus völlig unterschiedliche Löhne und Gehälter erhalten. Bildungsökonomen können z.B. nur etwa ein Drittel der Verdienstunterschiede auf unterschiedliche Bildungsniveaus zurückführen. Worauf die restlichen zwei Drittel zurückzuführen sind - auf unterschiedliche individuelle Anstrengungen, auf Glück oder Pech - ist unbekannt. Es liegt jedoch nahe, dem Glück oder Zufall eine große Rolle für unterschiedliche Erwerbs- und Einkommenschancen zuzuweisen. Solange dafür keine nachvollziehbaren Berechnungsgrundlagen zur Verfügung stehen, ist darüber hinaus anzunehmen, dass diese Zuweisung stark von kulturellen Einflüssen und von Persönlichkeitsmerkmalen abhängt.

Menschen müssen aber verantwortlich gemacht werden für Verteilungen, die Ergebnis ihrer Wahl sind, wie die persönliche Entscheidung, weniger zu arbeiten, bestimmte (exotische) Vorlieben zu haben oder einen bestimmten Beruf zu wählen. Wer den wenig riskanten Beruf eines Lehrers oder einer Lehrerin wählt, kann nicht mit Reichtum, aber mit einem gesicherten und stetigen Auskommen rechnen. Wer den riskanten Beruf eines Künstlers oder einer Künstlerin wählt, muss mit einer Achterbahn der Einkommensströme im Erwerbsverlauf rechnen, die in Armut oder Reichtum enden kann.

Folgt man Dworkin weiter, lässt sich dem „Diskurs des sozialen Risikomanagements“ das Prinzip der Verantwortungsethik unterstellen. Danach können Menschen für ihre Entscheidungen nur verantwortlich gemacht werden, wenn sieund das entspricht dem ethischen Prinzip der Solidarität - mit gleichen Ressourcen ausgestattet sind. Dworkin meint das sehr konkret. Da Einkommensverteilungen durch Zufall und externe Umstände bedingt sind, muss es innerhalb und zwischen den Generationen zu periodischen Umverteilungen kommen, beispielsweise durch hohe Erbschafts- und progressive Einkommensteuern. Diesem Gedankengang entspricht auch der von dem Yale-Ökonomen Robert Shiller (2003) entwickelte Vorschlag einer allgemeinen Versicherung gegen Ungleichheit: Das Steuersystem solle so umgestaltet werden, dass die Steuersätze sich an einer demokratisch entschiedenen und politisch festgelegten Verteilungsstruktur orientierten. ${ }^{4}$

\subsection{BEFÄHIGUNG ZU SELBST- BESTIMMTEM HANDELN ALS GERECHTIGKEITSGEBOT}

Problematisch ist Dworkins mangelnde Qualifizierung der Prämisse „gleicher Ressourcen“. Um welche Ressourcen geht es: materielle oder immaterielle, individuelle oder kollektive? Wann sind sie "gleich“ verteilt?: dem Bedürfnis, der Chance, dem Status (etwa Alter), der Leistung gemäß? Wann ist eine Präferenzäußerung Ausdruck einer freien und autonomen Wahl bzw. Entscheidung, deren Konsequenzen dann auch selbst zu verantworten wären? Alle mit diesen Fragen verbundenen Feinheiten können hier nicht diskutiert werden. ${ }^{5}$ Der im Hinblick auf eine Stärkung von Autonomie relevanteste Ansatz in dieser Debatte ist das Konzept der Fähigkeit oder besser der Befähigung („,capability“) von Amartya Sen (2001, 2009). Dieser Ansatz versucht nicht, das ontologische („letztwahre“) Fundament gerechter Institutionen weiter auszubauen, sondern geht pragmatisch vor und stellt die Folgen institutioneller Arrangements für Lebenschancen in den Vordergrund.

Für Sens Theorie der Befähigungsgerechtigkeit ist materielle Gleichheit allenfalls eine notwendige, aber keine hinreichende Bedingung für eine gerechte Verteilung von Lebenschancen. Es geht vielmehr um die individuelle Fähigkeit der Verwirklichung („functioning“), d.h. um die Fähigkeit, vorhandene Ressourcen in eigens gesetzte Ziele umzusetzen. Es geht also um eine dynamische Ressourcenausstattung, die individuell sehr ungleich verteilt sein kann, die aber jeder Person erlaubt, ihre eigenen Lebenspläne zu verwirklichen. Für eine solche funktionale Befähigung spielen, nach Auffassung von Sen, materielle Freiheiten eine große Rolle. Auf den Arbeitsmarkt bezogen bedeutet

4 Angenommen, die Verteilungsregel hieße, der Ginikoeffizient der Einkommensverteilung dürfe 0,33 nicht überschreiten; sobald das Kriterium überschritten wird, müssten die Einkommensteuer und die entsprechende Umverteilung (etwa über die Progression des Steuersatzes) solange angepasst werden, bis dieses Niveau wieder unterschritten wird.

5 Amartya Sens neuestes Buch "The Idea of Justice" (2009) ist ein Paradigma für solche feinziselierten Argumentationsstränge, spannend u.a. auch deswegen, weil es versucht, "östliches" und „westliches" philosophisches Gedankengut einander gegenüberzustellen und teilweise zu amalgamieren. 
dies die Freiheit, zwischen verschiedenen Berufen und Beschäftigungsformen wählen zu können, die Möglichkeit, Arbeit und Leben in Übereinstimmung ${ }^{6} \mathrm{zu}$ bringen, sowie die Möglichkeit, Fähigkeiten zu regenerieren oder zu erweitern.

Zur Befähigungsgerechtigkeit gehört aber auch der Zugang zu externen Ressourcen, wie eine funktionierende Nachbarschaft, eine ausgebaute öffentliche Infrastruktur, gleiche Bildungschancen, Partizipationsmöglichkeiten im politischen Gemeinwesen und im Betrieb, und - nicht zuletzt - ein funktionierender Arbeitsmarkt. Nur so können die Wahlfreiheit von Beruf und Beschäftigungsformen oder die Balance von Arbeit und Leben gewährleistet werden. Das Ziel kann nicht der magere Sozialstaat sein, der nur die Freiheit von unmittelbaren Bedürfniszwängen gewährleistet. Der moderne demokratische Sozialstaat sollte sich ein ehrgeizigeres Ziel setzen, nämlich die Freiheit zum Handeln, also die Befähigung zu einer eigenständigen und erfüllten Lebensführung.

Diese Freiheit - und hier trifft Sen auf Dworkins Verantwortungsethik - ist auf der Gegenseite jedoch mit der Verantwortung verbunden, für die Konsequenzen der getroffenen (freien) Wahl auch gerade zu stehen („accountability“). Denn Befähigung ist die Macht, etwas zu tun, und die Ausübung dieser Macht schafft Verantwortung, die unter Umständen auch mit Pflichten verbunden ist. ${ }^{7}$ So wie Eigentum im Sinne von befähigender Macht (kognitiver, physischer wie materieller Art) verpflichtet (vgl. auch Art. 14 Grundgesetz (GG)), so besteht dann freilich auch die Pflicht, Voraussetzungen für ein solches Eigentum auch zu schaffen.

Der alte deutsche Begriff „Erwerbsfähigkeit" könnte mit diesem Konzept wiederbelebt und um eine Entscheidungs- und Nachhaltigkeitsdimension erweitert werden. Im „erwerbsfähigen“ Alter (derzeit 20-64 Jahre), gesund und mit elementarer Bildung ausgestattet zu sein, genügt heute nicht mehr für eine autonome und nachhaltige Erwerbsfähigkeit im Lebenslauf. Nicht nur steigen die Anforderungen an den Grundstock der Bildung, der nötig ist, um erfolgreich ins Erwerbsleben einzutreten. Es steigt auch der Bedarf, diesen Grundstock im Erwerbsverlauf ständig zu erneuern oder zu ergänzen. Hinzu kommt noch die mögliche Einschränkung der Erwerbsfähigkeit durch Sorgepflichten, die kompensiert werden muss. Die damit im- plizit angesprochene Gender-Dimension bleibt in Amartya Sens Konzept unterbelichtet, wird aber beispielsweise von Martha Nussbaum $(2000,2003)$ aufgegriffen und von Jane Lewis und Susy Guillari (2005) weiterentwickelt. Sie plädieren für einen Katalog der Gleichverteilung von Grundfähigkeiten, die über den materiellen Ressourcenansatz hinausgehen und beispielsweise auch die Fähigkeit und Befähigung zu pflegenden, hinwendenden und erziehenden Tätigkeiten („care“) einschließen. Solange nicht auch Männer an diesen Tätigkeiten gleichermaßen teilhaben, ist die materielle Freiheit zur Berufswahl der Frauen eingeschränkt.

\subsection{PROBLEME DER EXKLUSION}

Schon das letzte Beispiel, die Einbeziehung der Gender-Dimension, zeigt die strukturelle Gefahr jeder (normativen) Spielregel gesellschaftlicher Ordnung: die Exklusion von Menschen, die nicht willens oder in der Lage sind, die Spielregeln zu erfüllen. Auf den Arbeitsmarkt bezogen heißt das ganz konkret: Wer gehört dazu? Wer ist in dieses „Spiel“ eingeschlossen? Frauen wurden beispielsweise lange ausgeschlossen, etwa durch die „Zölibatsregel“: Wenn Beamtinnen heirateten, mussten sie ihr Beschäftigungsverhältnis aufgeben, und noch bis 1976 verlangte das Bürgerliche Gesetzbuch die Zustimmung des Ehemannes, wenn seine Frau bezahlter Arbeit nachgehen wollte. Die Inklusionsfrage geht jedoch weit über die Genderfrage hinaus. Wer ist erwerbsfähig? Wer ist erwerbsberechtigt? Wer ist erwerbspflichtig? Wenn - wie mit dem Sozialgesetzbuch Zweites Buch (SGB II 2005) endgültig so beschieden - jede Person als erwerbsfähig zählt, die in der Lage ist, täglich regelmäßig wenigstens drei Stunden zu arbeiten, folgt aus dem Gerechtigkeitsprinzip sozialer Inklusion auch die gesellschaftliche Verpflichtung, die Arbeitsmarktspielregeln entsprechend anzupassen. Analog zur Pflicht der Vereinbarkeit von Familie und Beruf folgt daraus auch die Pflicht der Vereinbarkeit von Leistungsfähigkeit und Beruf. In anderen Worten: Nicht nur die Menschen sind fit für den Arbeitsmarkt zu machen, sondern auch der Arbeitsmarkt ist fit für die Menschen zu machen. Und es geht noch weiter: Was ist Arbeit? Nur bezahlte oder auch unbezahlte? Die Antwort ist heute eine andere als noch vor einer Generation: Nicht nur bezahlte, sondern auch jede unbezahlte, aber gesellschaftlich verpflichtende Tätigkeit (wie Erziehung und Pflege von Kindern) ist als Arbeit anzuerkennen (Supiot 2001, S. 54).

\section{3 \\ Gerechtigkeitsvorstellun- gen und Autonomie}

Wenn Freiheit also die Autonomie bedeutet, ein mit guten Gründen selbst gewähltes Leben führen zu können (und nicht allein von äußeren Zwängen und Notlagen getrieben zu sein), dann setzt dies soziale und materielle Ressourcen und Fertigkeiten im Umgang mit Ressourcen voraus, die Planungshorizonte eröffnen, indem sie Verlässlichkeit stiften. Nur diejenigen können Freiheit jenseits sozial-materieller Grundsicherheiten (oder gar im Gegensatz zu ihnen) imaginieren, die sich über Letztere keine Gedanken machen müssen.

Erforderlich für ein selbstbestimmtes Leben wäre Sen zufolge aber noch ein Weiteres: gesellschaftliche Verhältnisse nämlich, die es den Individuen ermöglichen, darüber gemeinsam zu entscheiden, was solche guten Gründe sind und wie bei der Aufteilung der notwendigen Ressourcen vorzugehen sei. Erst hier werden Gerechtigkeitsfragen auch zu Verteilungsfragen. Dagegen ist der Anspruch auf ein selbstbestimmtes Leben seinerseits nicht verhandelbar. Seine Missachtung, und das heißt: die Exklusion von Bevölkerungsgruppen, denen ein selbstbestimmtes Leben vorenthalten wird, bricht mit der Grundlage gesellschaftlichen Zusammenlebens.

Die hier angesprochene zweite Bedingung verbindet Sens Begriff von Freiheit (als Autonomie) wesentlich mit dem der Demokratie - Letztere aber nicht mehr nur als formales Verfahren der Beteiligung an Wahlen verstanden, sondern materiell gefüllt durch die realen Teilhabemöglichkei-

6 Zur Balance von Erwerbs- und Lebenswelt vgl. u.a. Gottschall (2010).

7 Dieser Punkt - oft übersehen - ist es wert, in einem Originalzitat belegt zu werden: „Freedom to choose gives us the opportunity to decide what we should do, but with the opportunity comes the responsibility for what we do - to the extent that they are chosen actions. Since a capability is the power to do something, the accountability that emanates from that ability - that power - is a part of the capability perspective, and this can make room for demands of duty - what can be broadly called deontological demands" (Sen 2009, S. 19). 
ten entscheidungsfähiger Menschen (erste Bedingung).

Dass die zweite Bedingung auch im Rahmen repräsentativer Demokratien in den hoch entwickelten kapitalistischen Gesellschaften der Gegenwart allenfalls partiell erfüllt wird, dürfte kaum strittig sein. Was für ein „menschenwürdiges Leben“ notwendig ist, wird in Deutschland gerade wieder nach politischer Opportunität entschieden, nicht danach, was in der Erfahrung der Bevölkerung unverzichtbare Güter, Dienstleistungen und Lebensumstände sind. Und ob es gerechtfertigt ist, dass die Hauptverursacher der Finanzmarkt- und Wirtschaftskrise, die Banken und sonstigen Finanzinstitute, am wenigsten zur Begleichung der Kosten dieser Krise herangezogen werden, darüber haben die Steuerzahler, die in die finanzielle Bresche springen mussten, erst gar nicht zu befinden. Nicht nur in diesem Fall durchkreuzt Marktmacht Demokratie.

Dennoch haben und behalten die Menschen ihre eigenen Gerechtigkeitsvorstellungen im Kopf. Gerechtigkeitstheoretiker, die sich nicht allein an philosophischen Reflexionen, sondern auch am empirisch fassbaren Gerechtigkeitssinn der Menschen orientieren, weisen darauf hin, dass es nicht nur einen Kompass der Gerechtigkeit, sondern mehrere gibt, mit deren Hilfe die Menschen jeweils unterschiedliche Aspekte ihrer sozialen Welt unterschiedlich beurteilen. David Miller's Hinweis, dass sich die jeweiligen Kriterien je nach sozialem Kontext unterscheiden, erscheint plausibel (Miller 2008, S. 66f.).

\subsection{ERGÄNZUNGEN UND KONFLIKTE ZWISCHEN GERECHTIGKEITS- VORSTELLUNGEN}

Wie David Miller argumentiert auch François Dubet, dass die verschiedenen Gerechtigkeitsprinzipien, die die Menschen in ihrem Alltag anwenden, immer wieder in Konflikt miteinander geraten können. Sie fügen sich nicht harmonisch ein in einen abgerundeten „Horizont der Gerechtigkeit", wirken in ihrer Pluralität vielmehr als ,praktisch notwendige Fiktionen“, die die Menschen pragmatisch auszubalancieren versuchen, ohne die aber gesellschaftliches Zusammenleben nicht möglich wäre. So steht Autonomie, für Dubet eines der wesentlichen Gerechtigkeitsprinzipien, im Spannungsverhältnis zur Gleichheit und Leistung, den beiden anderen im Bewusst- sein der Menschen verankerten, zentralen Richtwerten des Gerechtigkeitssinns (Dubet 2008, S. 482). Diese relative Eigenlogik von Gerechtigkeitsprinzipien, die sich aufeinander beziehen, aber auch partiell neutralisieren, könnte erklären helfen, warum zwar im letzten Jahrzehnt das empirisch erhobene Ungerechtigkeitsempfinden in Deutschland dramatisch zugenommen hat (Kronauer 2008, S. 372), sich bislang aber nicht in einem ebenso drastischen Loyalitätsentzug gegenüber den wirtschaftlichen und politischen Eliten bündelt.

Gerade wenn es darum gehen soll, Autonomie zu stärken, erscheint es deshalb notwendig, die Wechselbeziehungen und Spannungen zwischen den Gerechtigkeitsprinzipien zur Kenntnis zu nehmen, wie sie sich in den Wahrnehmungen der Menschen manifestieren. Denn hier, wenn überhaupt, sind die Anknüpfungspunkte für eine Politik des Sozialen zu verorten. Im Folgenden stützen wir uns insbesondere auf die bereits genannten Arbeiten von Miller und Dubet, verweisen aber auch auf den Beitrag von Becker (in diesem Heft).

\subsection{GRENZEN DES LEISTUNGS- PRINZIPS}

Miller (2008) hebt drei Gerechtigkeitsgrundsätze hervor, die er auch empirisch verankert sieht, ${ }^{8}$ und ordnet sie drei sozialen Kontexten zu:

- den Bedarfsgrundsatz der „solidarischen Gemeinschaft", die über unterschiedlich starke Loyalitäten hergestellt wird - sie können von intensiven Bindungen in der Familie bis zu den lockeren $\mathrm{Zu}$ gehörigkeitsformen zum nationalstaatlich verfassten Gemeinwesen reichen;

- den Leistungs- oder Verdienstgrundsatz dem „Zweckverband“, der in erster Linie (aber nicht nur) über ökonomische Beziehungen gestiftet wird;

- den Gleichheitsgrundsatz der „Staatsbürgerschaft“, wobei zur Sicherstellung von staatsbürgerlicher Gleichheit soziale Rechte unabdingbar sein können (ebd., S. 66ff.).

Es soll hier nicht um die Plausibilität dieser Abgrenzungen gehen. Interessant im Hinblick auf unser Thema ist vielmehr, wie sich die drei Prinzipien (und die ihnen zugrunde liegenden oder auch nur zugrunde gelegten sozialen Kontexte) zueinander verhalten und welche Folgerungen sich da- raus für die Ermöglichung selbstbestimmten Handelns ergeben.

Autonomie selbst steht bei Miller nicht ausdrücklich zur Diskussion. Das dürfte seinen Grund darin haben, dass er sich auf Fragen der Verteilungsgerechtigkeit konzentriert. Die grundlegendere Frage nach den Voraussetzungen eines selbstbestimmten Handelns ist nachrangig, aber gleichwohl als Hintergrund präsent. Dies wird am deutlichsten in der Behandlung des Leistungsgrundsatzes.

Hier argumentiert Miller zunächst als Verfechter der meritokratisch (auf Grund von Leistungen) gerechtfertigten Ungleichheiten. Der Markt gilt ihm dabei als mehr oder weniger verlässlicher Lieferant von Bewertungen unterschiedlicher Leistungen. ${ }^{9}$ Zwar räumt er Schwierigkeiten bei der Zuordnung kollektiv erbrachter Leistungen zu Individuen ein, sieht darin aber keinen grundsätzlichen Einwand. Leistungsgerechtigkeit zeigt sich hier im Konflikt mit dem Gleichheitsgrundsatz. Wo aber liegen die Grenzen des meritokratischen Maßstabs? Zunächst einmal in der - um mit Sen zu sprechen „mit guten Gründen" - getroffenen gesellschaftlichen Entscheidung darüber, welche Leistungen dem Markt entzogen sein müssen, um allgemein verfügbar zu sein - in der Entscheidung über meritorische (allen zu gewährleistende) Güter wie Bildung und Gesundheitsversorgung also, die nach ihrem eigenständigen „Wert“ zur Wohlfahrt unverzichtbar sind. Hier kommen andere Leistungsmaßstäbe ins Spiel als sie vom Markt honoriert werden. Sodann und vor allem aber liegen für Miller die Grenzen des Leistungsgrundsatzes in der Herrschaft des Zufalls (man könnte auch genauer sagen: der Herrschaft der Konkurrenz), der dem Marktmechanismus immanent ist. „Wenn alle diese Vergünstigungen indirekt nach dem Leistungsprinzip zugewiesen werden, leben wir in einem System, in dem viel zu viel davon abhängt, ob wir zufällig in der Lage sind, genau die Güter herzustellen, für die der Markt oder der Staat zu zahlen bereit sind. Unter diesen Bedingungen hat meine Verteidigung der Meritokratie ernsthafte Schwächen“ (Miller 2008, S. 251).

8 Auf methodische Probleme bei Millers Vorgehen weist Honneth in seinem Vorwort (ebd.) hin; darauf können wir hier allerdings nicht eingehen.

9 Wieder würde es den Rahmen dieses Textes sprengen, auf die Argumente im Einzelnen einzugehen, auch dort, wo sie durchaus Kritik herausfordern. 
In gewisser Hinsicht überschneiden sich die Prinzipien von Leistung und Autonomie. Schließlich verheißt das Leistungsprinzip Selbstbestimmung über das eigene Leben aufgrund eigener Fähigkeiten und Anstrengungen. Es lässt sich am Markt jedoch allenfalls partiell und nur unter glücklichen Umständen realisieren. Unter den Bedingungen einer Marktgesellschaft ist deshalb Autonomie bereits im Blick auf die Zurechenbarkeit der Folgen des eigenen Handelns immer eingeschränkt und gefährdet. Aus den Grenzen des Leistungsprinzips folgert Miller, dass ihm ein Gegengewicht zur Seite stehen muss: „Der entscheidende Punkt dabei ist, dass Meritokratie generell akzeptabel ist, wenn sie durch Prinzipien und Institutionen ausgeglichen wird. Dafür muss staatsbürgerliche Gleichheit herrschen, die den Menschen unabhängig von irgendeiner Leistung einen sozialen Status und eine Reihe handfester Vergünstigungen sichert. Außerdem sollte dafür gesorgt werden, dass Leistungen auf einem Gebiet durch Leistungen auf einem anderen Gebiet aufgewogen werden können“ (Miller 2008, S. 252). Ein selbstbestimmtes Leben ist somit überhaupt nur unter der Voraussetzung denkbar, dass Marktabhängigkeit durch staatsbürgerlich gewährleistete Gleichheiten und Sicherheiten konterkariert wird. ${ }^{10}$

\subsection{AUTONOMIE ALS LEITIDEE VON GERECHTIGKEITSVORSTELLUNGEN}

Für Dubet stellt Autonomie zunächst ein eigenständiges Gerechtigkeitsprinzip neben Gleichheit ${ }^{11}$ und Leistung dar. In seiner Studie ,zum subjektiven Ungerechtigkeitsempfinden am Arbeitsplatz", so der Untertitel der deutschen Ausgabe, bemisst sich Autonomie vornehmlich an den Möglichkeiten der Selbstverwirklichung in der Arbeit. Dazu gehört genuin die „Fähigkeit, sein Leben selbst zu meistern“, „das Leben nach seinen eigenen Vorstellungen zu gestalten". Hier bezieht sich Dubet ausdrücklich auf Sen (Dubet 2008, S. 31).

Der Autonomie kommt aber noch ein weiterer, umfassenderer Stellenwert in dem von Dubet dargelegten sozialen Kosmos der Gerechtigkeitsvorstellungen zu als nur der eines einzelnen Prinzips, das zu den beiden anderen in einem spannungsreichen Verhältnis von Ergänzung und Konflikt steht. „Wenn es keine absolut gerechte Welt gibt, an der sich die Kritik und das Handeln ausrichten können, wenn wir dazu verurteilt sind, widersprüchliche Prinzipien zu kombinieren, dann muss sich der Gegenstand der Gerechtigkeit auf das Individuum verlagern. Die größte Ungerechtigkeit ist dann diejenige, die das Individuum zerstört, die es daran hindert, Subjekt seines Lebens zu sein, die es ihm verbietet, sich angesichts oder trotz der Ungerechtigkeiten zu entwickeln" (Dubet 2008, S. 488).

In diesem Sinn wird Autonomie gewissermaßen zum Ankerpunkt aller sozialen Ungerechtigkeitserfahrungen und Gerechtigkeitsvorstellungen, ihre Stärkung folgerichtig zum vordringlichen Ziel: „Da das Individuum gleichzeitig Realisierung eines Sozialisationsprogramms, kognitiver Apparat und Wille zur Autonomie ist, müssen wir erkennen, wie es ihm gelingen kann, Subjekt zu sein. Und eine gute Arbeitsorganisation wie auch eine gute Gesellschaft ist die, die es bei dieser Arbeit unterstützt. Daraus folgt, die Sozialpolitik $\mathrm{zu}$ individualisieren, die Arbeitenden mit einem Beruf und mit Kompetenzen auszurüsten, ihre Bildung zu verbessern, ihnen Flexibilität zu ermöglichen, ohne dass sie dabei ihr Selbst verlieren - kurz, die Tatsache ernst zu nehmen, dass wir in einer Gesellschaft von Individuen leben“ (Dubet 2008, S. 489). Was dies für eine „verhaltenssensible Arbeitsmarktpolitik" bedeuten könnte, umreißt Günther Schmid (in diesem Heft). Allerdings ist Dubets Rede von einer "Gesellschaft von Individuen“ überaus voraussetzungsvoll. Denn sie unterstellt, wie wir zuvor gezeigt haben, bereits grundlegende materielle Gleichheiten und Sicherheiten, die die Entfaltung von Individualität erst ermöglichen.

\subsection{AUTONOMIE UND GESELL- SCHAFTLICHE TEILHABE (INKLUSION)}

Die Studien von Miller und Dubet, gestützt auf empirische Erhebungen zu Gerechtigkeitsvorstellungen, schlagen eine wichtige Brücke zu einer bereits angesprochenen, verwandten gesellschaftspolitischen Diskussion, der über Inklusion und Exklusion. Exklusion als Verweigerung gesellschaftlicher Teilhabe bedeutet zugleich die schärfste Verweigerung von Autonomie. Wie an anderer Stelle ausgeführt, sind es vor allem drei Instanzen, die in den hoch entwickelten kapitalistischen Gesellschaften der Gegenwart Zugehörigkeit und Teilhabe vermitteln oder verhindern:
- die Vergesellschaftung durch Arbeit, was gegenwärtig heißt, in der vorherrschenden Form der Erwerbsarbeit;

- die Einbeziehung in die Wechselseitigkeiten, die durch soziale Nahbeziehungen gestiftet werden;

- der Zugang zu und die Realisierung von persönlichen, politischen und sozialen Rechten, die eine Partizipation am Lebensstandard und an den Lebenschancen ermöglichen, wie sie in einer gegebenen Gesellschaft historisch erreicht und angelegt sind.

Die verschiedenen Instanzen zeichnen sich durch unterschiedliche Zugangslogiken zu und Zuteilungslogiken von Teilhabemöglichkeiten aus. Sie ergänzen einander, aber auf widersprüchliche Weise. Ihr Zusammenwirken und damit ihre Inklusionskapazität ist an entscheidender Stelle gefährdet: an der marktförmigen Organisation der Arbeitsteilung, die ein Machtgefälle zwischen Kapital und Arbeit einschließt und damit zugleich die Möglichkeit der Verweigerung eines sozialen Rechts auf Arbeit (Kronauer 2010, S. 231f.).

Die relative Eigenständigkeit der Instanzen, die auf je eigene Weise Zugehörigkeit und Teilhabe vermitteln, findet sich in der relativen Eigenständigkeit der Gerechtigkeitsgrundsätze, wie Miller sie unterscheidet, wieder. Und wie es beim Ziel einer Stärkung der individuellen Autonomie im Sinne Dubets darauf ankommt, unterschiedliche Gerechtigkeitsprinzipien zugleich aushalten und verfolgen zu können, so kommt es auch bei einer Politik des Sozialen, die die gesellschaftlichen Voraussetzungen von Autonomie stärken soll, darauf an, den jeweils besonderen Beiträgen der verschiedenen Inklusionsinstanzen Rechnung zu tragen (Kronauer 2010, S. 262f.). Was heißt das?

10 Diese Einsicht deckt sich mit der (oben dargestellten) Sichtweise von Ronald Dworkin, wonach Bedarf nach periodischer Umverteilung besteht, um die durch Zufallsgesetze hervorgerufene Ungleichheit grundlegender Ressourcen wiederherzustellen.

11 Gleichheit hat bei Dubet allerdings eine andere Konnotation als bei Miller. Gemeint sind für legitim gehaltene Formen der Ungleichheit, die es erlauben, darüber zu urteilen, was nach dem Prinzip "jedem das Seine“ jedem zusteht. Diese legitimen Formen der Ungleichheit setzen Gleichheit insofern voraus, als ein für alle bindendes gesellschaftliches Einverständnis über die legitimen Prinzipien der Positionsverteilung unterstellt wird 
4

Nach den zuvor dargelegten Überlegungen über die Grenzen des marktvermittelten Leistungsprinzips, das Gebot von Chancen- und Ressourcengleichheit und die Befähigung zu selbstbestimmtem Handeln als Voraussetzungen von Demokratie setzt eine Stärkung von Autonomie Gleichheiten und Sicherheiten in allen angesprochenen wesentlichen Dimensionen gesellschaftlicher Teilhabe voraus:

(1) Es gilt die Unabhängigkeit aller Bürgerinnen und Bürger von den Wechselfällen des Marktes zu sichern - durch den marktunabhängigen, gleichen und sozial ausgleichenden Zugang zu meritorischen Gütern, darunter insbesondere Bildung und Gesundheit, sowie eine kulturell angemessene Mindestsicherung des Lebensstandards über den Lebensverlauf hinweg (siehe die Beiträge von Böhnke, Becker und Hopf in diesem Heft).

(2) Es gilt darüber hinaus, die Handlungs- und Gestaltungsspielräume in der Erwerbsarbeit durch neue Formen der in- dividuellen Absicherung in der Arbeit und am Arbeitsmarkt (siehe die Beiträge von Lohmann/Andreß und Schmid in diesem Heft) sowie der Partizipation bei Entscheidungen des Managements zu erweitern.

(3) Schließlich gilt es, die individuellen Handlungs- und Gestaltungsmöglichkeiten außerhalb der Erwerbsarbeit durch die zeitliche, materielle und soziale Anerkennung nicht-erwerbsförmiger Tätigkeiten und eine gleichberechtigte und -verpflichtende Arbeitsteilung zwischen den Geschlechtern zu stärken.

Warum muss es ein zentrales gesellschaftspolitisches Ziel sein, ein selbstbestimmtes Leben für alle zu ermöglichen? Weil davon die Lebensqualität in der Gesellschaft und ihre demokratische Qualität abhängt. Die zunehmende Marktabhängigkeit in fast allen gesellschaftlichen Bereichen verbreitet das Gift der Unsicherheit, in unterschiedlich starken Dosen, durch die gesamte Gesellschaft. Unsicherheit schränkt die Möglichkeiten, ein selbstbestimmtes Leben zu führen, grundlegend ein. Am stärksten sind davon diejenigen betroffen, die der Marktabhängigkeit am direktesten ausgeliefert sind. Aber auch in den abhängig beschäftigten Mittelklassen machen sich Unsicherheiten und Verunsicherungen breit. Diesen Mittelklassen wird es nicht besser gehen, wenn es den (arbeitenden und arbeitslosen) Armen noch schlechter geht. Nutznießer sind vielmehr diejenigen, die mit Marktmacht zugleich gesellschaftliche und politische Macht ausüben können. Ihnen reden die Elitisten, die Verächter der „Plebs“ und Verfechter der Ungleichheit, das Wort.

Die anschließenden Beiträge stimmen darin überein, dass Autonomie, soll sie verallgemeinerungsfähig sein, grundlegender gesellschaftlicher Gleichheiten und Sicherheiten bedarf. Sie weisen empirisch die Grenzen einer Leistungsgerechtigkeit nach, die auf Bestätigung am Markt setzt; sie zeigen, wie eine gesellschaftliche Beschränkung von Ungleichheit sowie verlässliche Institutionen der sozialen Sicherung die Möglichkeiten zur individuellen Selbstbestimmung und die Lebensqualität in einer Gesellschaft insgesamt steigern; sie arbeiten die Bedeutung realer Chancengleichheit im Bildungssystem heraus, sowohl für den Wettbewerb um Bildungstitel als auch für das wesentliche Bildungsziel der Vermittlung von gesellschaftlicher Teilhabe. Und sie zeigen Wege zu neuen Formen der Absicherung am Arbeitsmarkt auf, die die Autonomie der abhängig Beschäftigten stärken können.

\section{LITERATUR}

Arneson, R. J. (2010): Luck Egalitarianism - A Primer, in: Knight, C./ Stemplowska, Z. (Hrsg): Responsibility and Distributive Justice, Oxford, S. $138-161$

Becker, I./Hauser, R. (2009): Soziale Gerechtigkeit - ein magisches Viereck. Zieldimensionen, Politikanalysen und empirische Befunde, Berlin Bohrer, K.-H. (2009): Lobhudeleien der Gleichheit, in: Frankfurter Allgemeine FAZ.NET vom 21.10

Dubet, F. (2008): Ungerechtigkeiten, Hamburg

Dworkin, R. (2000): Sovereign Virtue - The Theory and Practice of Equality, Cambridge, MA/London

Gottschall, K. (2010): Arbeit, Beschäftigung und Arbeitsmarkt aus der Genderperspektive, in: Böhle, F./Voß, G./Wachtler, G.(Hrsg.): Handbuch für Arbeitssoziologie, Wiesbaden, S. 671-698

Kronauer, M. (2008): Verunsicherte Mitte, gespaltene Gesellschaft?, in: WSI-Mitteilungen 61 (7), S. 372-378
Kronauer, M. (2010): Exklusion. Die Gefährdung des Sozialen im hoch entwickelten Kapitalismus, 2. akt. u. erweit. Aufl., Frankfurt am Main/ New York

Lewis, J./Giullari, S. (2005): The Adult Worker Model Family, Gender Equality and Care: The Search for New Policy Principles and the Possibilities and Problems of a Capabilities Approach, in: Economy and Society 34 (1), S. 76-104

Miller, D. (2008): Grundsätze sozialer Gerechtigkeit, Frankfurt am Main/New York

Nussbaum, M. (2000): Women and Human Development. The Capabilities Approach, Cambridge

Nussbaum, M. (2003): Capabilities as Fundamental Entitlements: Sen and Social Justice, in: Feminist Economics 9 (2-3), S. 33-59

Rawls, J. (1990): A Theory of Justice, Oxford, (first published 1970) 
Rawls, J. (2001): Justice as Fairness - A Restatement (edited by Erin Kelly), Cambridge/London

Roemer, J. E. (2009): Equality: Its Justification, Nature, and Domain, in: Salverda, W./Nolan B./Smeeding T. S. (Hrsg.): The Oxford Handbook of Economic Inequality, Oxford, S. 23-39

Salais, R. (2003): Work and Welfare: Toward a Capability Approach, in: Zeitlin, J./Trubek, D. M. (Hrsg): Governing Work and Welfare in a New Economy. European and American Experiments, Oxford, S. 317-344 Sarrazin, T. (2009): Klasse statt Masse. Von der Hauptstadt der Transferleistungen zur Metropole der Eliten. Thilo Sarrazin im Gespräch, in: Lettre International (86), S. 197-201

Schmid, G. (2008): Full Employment in Europe - Managing Labour Market Transitions and Risks, Cheltenham, England/Northampton, MA, USA

Schmid, G. (2011): Übergänge am Arbeitsmarkt: Arbeit, nicht nur Arbeitslosigkeit versichern, Berlin
Sen, A. (2001): Development as Freedom, New York [Deutsch: Ökonomie für den Menschen, München (2002)]

Sen, A. (2009): The Idea of Justice, London

Shiller, R. J. (2003): The New Financial Order: Risk in the 21st Century, Princeton

Sloterdijk, P. (2009): Das elfte Gebot: die progressive Einkommenssteuer, in: Frankfurter Allgemeine Zeitung (FAZ) vom 27.9., http://www.faz. net/s/RubCF3AEB154CE64960822FA5429A182360/Doc ECBEDDC9B 25CD497DA85BEBF7625D0483 ATpl Ecommon Scontent.html Supiot, A. (2001): Beyond Employment - Changes in Work and the Future of Labour Law in Europe, Oxford

Wilkinson, R./Pickett, K. (2009): The Spirit Level. Why More Equal Societies Almost Always Do Better, Harlow [Deutsch: Gleichheit ist Glück - Warum gerechte Gesellschaften für alle besser sind, Berlin (2009)] 